\title{
CLINICAL, ULTRASONOLOGICAL, PATHOLOGICAL CORRELATION OF BREAST MASSES
}

\author{
Megha Mittal1, Gaurav Agrawal2, Amit Agrawal'3, K. K. Agrawal', S. B. L. Shrivastav5, Mohini Shrivastav6, Pankaj Goyal7, \\ Priyanshi Mishra ${ }^{8}$
}

${ }_{1}^{1}$ Assistant Professor, Department of Radiodiagnosis, GR Medical College, Gwalior.

${ }^{2}$ Consultant, Department of Surgical Oncology, BIMR Oncology Centre, Gwalior.

${ }^{3}$ Resident, Department of Radiotherapy, Shri Ram Murti Smarak Institute of Medical Sciences, Bareilly.

${ }^{4}$ Consultant, BIMR Oncology Centre, Gwalior.

${ }^{5}$ Gynaeconcologist, BIMR Oncology Centre, Gwalior.

${ }^{6}$ Gynaecologist, BIMR Oncology Centre, Gwalior.

${ }^{7}$ Pathologist, BIMR Oncology Centre, Gwalior.

${ }^{8}$ Counsellor, BIMR Oncology Centre, Gwalior.

\section{ABSTRACT}

\section{QUESTIONS TO BE ANSWERED}

In this retrospective study we have correlated the clinical, ultrasonological and pathological findings of breast masses to answer whether we could rely on ultrasound and Fine Needle Aspiration (FNAC) findings for the further management of patients.

\section{MATERIAL AND METHODS}

This is a retrospective study done at Vidya Cancer Hospital, Gwalior, Madhya Pradesh, India. This study has included patients from Jan. 2014 to Jan. 2016. The total number of patients is 50. The clinical examination is done by an oncology team which includes a surgical oncologist, medical oncologist, radiation oncologist and gynaecologist. The ultrasound is done by an oncoradiologist. The pathological examination is done by an oncopathologist. The ultrasonology findings assessment is done according to the American College of Radiology, Breast Imaging Reporting and Data System (BI-RADS). The statistical analysis is done by using standard formulas.

\section{DISCUSSION}

In our day-to-day practice, we often see so many breast lumps. All the patients and their family members are in great agony. We as clinicians have to give quick results by non-invasive methodology. In today's era nothing could be advocated merely on clinical experience, it has to be evidence based. To create evidence, we have done this study. We found even a good clinical examination and ultrasound by a good team could solve most of the diagnostic dilemmas of breast masses. In our study, the sensitivity and specificity of clinical examination were $100 \%$ and $88 \%$ respectively. We tried to compare it with other studies, but we were unable to compare it with because of wide variations in those studies for they were either not focusing on clinical examination or it was not a team effort. Yes, we have been able to compare the sensitivity and specificity of ultrasound and FNAC findings. In our study, the sensitivity and specificity of ultrasound was $100 \%$ and $88 \%$ respectively which was comparable to $95.7 \%$ and $89.2 \%$ respectively in Lehman et al Study. In our study the sensitivity and specificity of FNAC was $93.3 \%$ and $88 \%$ respectively, which was $66.6 \%$ and $81.8 \%$ in Homesh NA et al study. The difference in sensitivity and specificity may be because our pathologist was always in coordination with the clinicians and ultrasonologist. Apart from this, we came across very interesting finding that our pathologist was not able to give any conclusive findings in clinically suspicious an $d$ BIRADS 4 findings.

\section{CONCLUSION}

This study has shown that a good team work could do wonders and a good clinical and ultrasonological examination could sort out most of the diagnostic dilemmas of breast masses. FNAC does well in frankly benign and malignant lesions. Any lesion which is suspicious and BIRADS 4 should undergo an upfront biopsy rather than FNAC. Fallacy was the number of patients included in this study was too small to make a final remark and secondly it is not a blinded study.

\section{KEYWORDS}

Breast, USG, FNAC

HOW TO CITE THIS ARTICLE: Mittal M, Agrawal G, Agrawal A, et al. Clinical, ultrasonological, pathological correlation of breast masses. J. Evolution Med. Dent. Sci. 2016;5(68):4864-4867, DOI: 10.14260/jemds/2016/1106

\section{INTRODUCTION}

Breast disease is now the leading disease in women worldwide. The large number of biopsies performed for benign breast abnormalities has long been recognized as a serious problem.(1) Excessive biopsies for benign lesions have adverse effects on society and on the women who undergo them by increasing the costs of screening projects causing

Financial or Other, Competing Interest: None

Submission 07-04-2016, Peer Review 25-05-2016,

Acceptance 02-06-2016, Published 23-08-2016.

Corresponding Author:

Dr. Megha Mittal,

Opposite Old High Court,

Lashkar.

E-mail:drmeghamittal@gmail.com

DOI: $10.14260 /$ jemds/2016/1106 morbidity and adding to the barriers that keep women from using a potentially life-saving procedure.(1-3) Attempts have been made to increase the positive predictive value for biopsy (Biopsy yield of cancer) by performing a complete diagnostic work-up that often includes Ultrasonography (US).

Refinement of high-frequency technology, particularly with 7.5-13 MHz probes, has brought out a totally new facet in

\section{USG breast Imaging.(2) For example:}

- High-density probes provide better lateral resolution.

- Harmonic imaging leads to improved resolution and reduced reverberation and near-field artifacts.

- Real-time compound scanning results in increased tissue contrast resolution. 
- Extended or panoramic views provide a better perspective of the lesion in relation to the rest of the breast.

Harmonic imaging and real-time compounding has been shown to improve image resolution and lesion characterisation. ${ }^{(3,4)}$ More recently, USG elastography seems to be quite promising. Initial results indicate that it can improve the specificity and positive predictive value of USG in the characterisation of breast masses. (5)

The reason why any lesion is visible on mammography or USG is the relative difference in the density and acoustic impedance of the lesion respectively as compared to the surrounding breast tissue.

This is exemplified in women with dense breast tissue, where USG is useful in detecting small breast cancers that are not detected on mammography.(6)

\section{Questions to be answered}

In this retrospective study we have correlated the clinical, ultrasonological and pathological findings of breast masses to answer whether we could rely on ultrasound and Fine Needle Aspiration (FNAC) findings for further management of patients.

\section{MATERIAL AND METHODS}

This is a retrospective study done at Vidya Cancer Hospital, Gwalior, Madhya Pradesh, India. This study has included patients from Jan. 2014 to Jan. 2016.

The total number of patients is 50 .

The clinical examination is done by an oncology team which includes a surgical oncologist, medical oncologist, radiation oncologist and gynaecologist. The ultrasound is done by an oncoradiologist. The pathological examination (FNAC and histopathology) is done by an oncopathologist.

The ultrasonology findings assessment is done according to the American College of Radiology Breast Imaging Reporting and Data System (BI-RADS).

The statistical analysis is done by using standard formulas.

\section{Analysis of Data}

In our study as depicted in Table 1, on evaluation of 50 breast masses clinically we found 25 lesions as benign, 3 lesions as suspicious and 22 lesions as malignant. Our ultrasonologist reported these lesions as BIRADS 3 in 25 lesions, BIRADS 4 in 3 lesions, BIRADS 5 in 22 lesions. Our pathologist on FNAC reported ductal carcinoma in 22 patients, fibroadenoma in 14 patients, fibroadenosis in 3 patients, inconclusive in 5 patients, abscess in 2 patients, cyst in 1 patient, galactocele in 2 patients and atypical ductal cells in 1 patient.

The histopathology report was positive for ductal carcinoma in 24 patients, extensive ductal carcinoma in 1 patient, fibroadenoma in 14 patients, cystosarcoma phyllodes in 1 patient.

We correlated the clinical, ultrasonological and pathological data. The 25 benign lesions on clinical examination were reported as BIRADS 3, 3 suspicious lesions were reported as BIRADS 4 and 22 malignant lesions were reported as BIRADS 5 .

We found FNAC results in favour of ductal carcinoma in 22 patients, which were clinically malignant and BIRADS 5. Five patients were reported as inconclusive, 3 patients out of 5 were clinically suspicious and BIRADS 4, 2 were benign and BIRADS 3. Twenty three patients were in favour of benign pathology and they were clinically benign and ultrasonologically BIRADS 3.

For calculating the sensitivity and specificity, we have excluded the cases in which histopathology was not done.

The sensitivity and specificity of clinical examination was $100 \%$ and $88 \%$ respectively. The sensitivity and specificity of ultrasound was $100 \%$ and $88 \%$ respectively. The sensitivity and specificity of FNAC was $93.3 \%$ and $88 \%$ respectively.

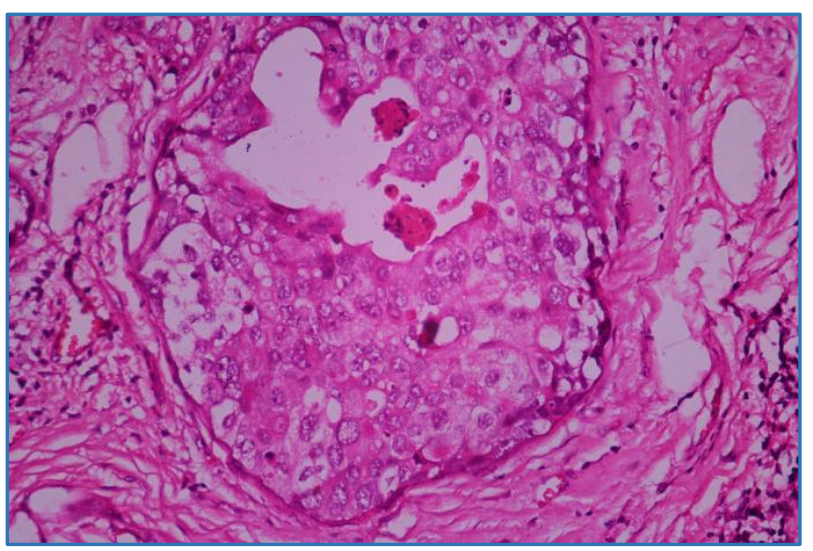

Fig.1: Ductal carcinoma in situ

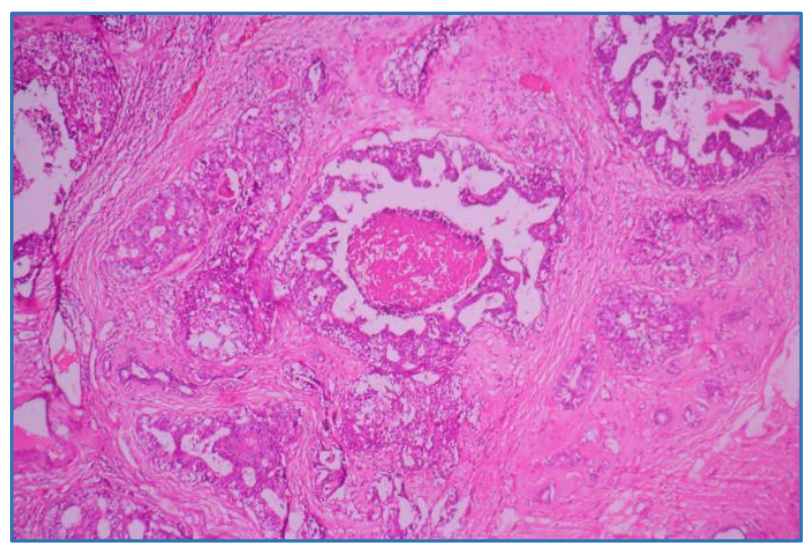

Fig.2: Ductal carcinoma in situ

\section{DISCUSSION}

In our day-to-day practice, we often see so many breast lumps. All the patients and their family members are in great agony. We as clinicians have to give quick results by non-invasive methodology. In today's era nothing could be advocated merely on clinical experience, it has to be evidence based. To create evidence, we have done this study.

We found even a good clinical examination and ultrasound by a good team could solve most of the diagnostic dilemmas of breast masses.

We have used the BIRADS system for ultrasound reporting, which is considered as gold standard for reporting breast masses. It has six categories.

\section{Category 0}

Incomplete: Need Additional Imaging Evaluation and/or Prior Mammograms for Comparison.

\section{Category 1}

Negative: There is nothing to comment on. This is a normal examination. 


\section{Category 2}

Benign: Like category 1, this is a normal assessment, but here the interpreter chooses to describe a benign finding in the mammography report. Involuting calcified fibroadenomas, skin calcifications, metallic foreign bodies (Such as core biopsy and surgical clips) and fat-containing lesions (Such as oil cysts, lipomas, galactocoeles and mixed-density hamartomas) all have characteristically benign appearances and may be described with confidence. The interpreter may also choose to describe intramammary lymph nodes, vascular calcification, implants or architectural distortion clearly related to prior surgery while still concluding that there is no mammographic evidence of malignancy. On the other hand the interpreter may choose not to describe such findings, in which case the examination should be assessed as negative (Category 1).

Note that both category 1 and category 2 assessments indicate that there is no mammographic evidence of malignancy. Both should be followed by the management recommendation for routine mammography screening. The difference is that category 2 should be used when describing one or more specific benign mammographic findings in the report, whereas category 1 should be used when no such findings are described (Even if such findings are present).

\section{Category 3}

Probably Benign: A finding assessed using this category should have a $\leq 2 \%$ likelihood of malignancy, but greater than the essentially $0 \%$ likelihood of malignancy of a characteristically benign finding. A probably benign finding is not expected to change over the suggested period of imaging surveillance, but the interpreting physician prefers to establish stability of the finding before recommending management limited to routine mammography screening. ACR BI-RADS® ATLASMAMMOGRAPH.

\section{Category 4}

Suspicious: This category is reserved for findings that do not have the classic appearance of malignancy, but are sufficiently suspicious to justify a recommendation for biopsy. The ceiling for category 3 assessment is a $2 \%$ likelihood of malignancy and the floor for category 5 assessment is $95 \%$, so category 4 assessments cover the wide range of likelihood of malignancy in between. Thus, almost all recommendations for breast interventional procedures will come from assessments made using this category.

\section{Category 5}

Highly suggestive of malignancy: These assessments carry a very high probability ( $\geq 95 \%$ ) of malignancy. This category initially was established to involve lesions for which 1-stage surgical treatment was considered without preliminary biopsy, in an era when preoperative wire localisation was the primary breast interventional procedure. Nowadays, given the widespread acceptance of imaging-guided percutaneous biopsy 1-stage surgery is rarely, if ever, performed. Rather, current oncologic management almost always involves tissue diagnosis of malignancy via percutaneous tissue sampling to facilitate treatment options, such as when sentinel node biopsy is included in surgical management or when neoadjuvant chemotherapy is administered prior to surgery. Therefore, the current rationale for using a category 5 assessment is to identify lesions for which any non-malignant percutaneous tissue diagnosis is automatically considered discordant resulting in the recommendation for repeat (usually surgical) biopsy.

\section{Category 6}

Known Biopsy-Proven Malignancy: This category is reserved for examinations performed after biopsy proof of malignancy (Imaging performed after percutaneous biopsy, but prior to complete surgical excision), in which there are no mammographic abnormalities other than the known cancer that might need additional evaluation.

In our study, the sensitivity and specificity of clinical examination were $100 \%$ and $88 \%$ respectively. We tried to compare it with other studies, but we were unable to do so because of wide variations in those studies for they were either not focusing on clinical examination or it was not a team effort.

Yes, we have been able to compare the sensitivity and specificity of ultrasound and FNAC findings. In our study the sensitivity and specificity of ultrasound was $100 \%$ and $88 \%$ respectively, which was comparable to $95.7 \%$ and $89.2 \%$ respectively in Lehman et al study.(7)

In our study the sensitivity and specificity of FNAC was $93.3 \%$ and $88 \%$ respectively, which was $66.6 \%$ and $81.8 \%$ in Homesh NA et al study.(8) The difference in sensitivity and specificity may be because our pathologist was always in coordination with the clinicians and ultrasonologist.

Apart from this, we came across a very interesting finding that our pathologist was not able to give any conclusive findings in clinically suspicious and BIRADS 4 findings.

\section{CONCLUSION}

This study has shown that a good team work could do wonders and a good clinical and ultrasonological examination could sort out most of the diagnostic dilemmas of breast masses.

FNAC does well in frankly benign and malignant lesions.

Any lesion which is suspicious and BIRADS 4 should undergo an upfront biopsy rather than FNAC.

Fallacy was that the number of patients included in this study was too small to make a final remark and secondly it is not a blinded study.

\section{REFERENCES}

1. World Health Organization Fact sheet No 297: Cancer, 2006.

2. Catarazi S, Guispetti GM, Rissato G, et al. Studio multicentrico per la valutazione della efficacia diagnostics della mammografia e della ecografia nelle neoplasie mammarie non palpabili. Radiol Med 1992;84(3):193-7.

3. Meritt CRB. Technology update. Radiol Clin North Am 2001;39:385-97.

4. Shapiro RS, Wagreich J, Parsons RB, et al. Tissue harmonic imaging sonography, evaluation of image quality compared with conventional sonography. AJR 1998;171(5):1203-6.

5. Zhi $\mathrm{H}, \mathrm{Ou}$ B, Luo BM, et al. Comparison of ultrasound elastography, mammography, and sonography in the diagnosis of solid breast lesions. J Ultrasound Med 2007;26(6):807-15.

6. Crystal P, Strano SD, Shcharynski S, et al. Using sonography to screen women with mammographically dense breasts. AJR Am J Roentgenol 2003;181(1):177-82. 
7. Lehman CD, Lee CI, Loving VA, et al. Accuracy and value of breast ultrasound for primary imaging evaluation of symptomatic women 30-39 years of age. AJR Am J Roentgenol 2012;199(5):1169-77.
8. Homesh NA, Issa MA, El-Sofiani HA. The diagnostic accuracy of fine needle aspiration cytology versus core needle biopsy for palpable breast lumps. Saudi Med J 2005;26(1):42-6. 